\title{
DISPLACEMENTS OF THE MENISCI UNDER JOINT LOAD: AN IN VITRO STUDY IN HUMAN KNEES
}

\author{
Donita I. Bylski-Austrow,* Michael J. Ciarelli, Dennis C. Kayner, \\ LARRY S. MATTHEWS and STEVEN A. GoldSTEIN \\ Orthopaedic Research Laboratories, Section of Orthopaedic Surgery, The University of Michigan, Ann \\ Arbor, MI 48109-0486, U.S.A.
}

\begin{abstract}
The purpose of this study was to test whether the menisci displace under joint compression combined with internal-external torques and anterior-posterior forces at fixed flexion angles. We further determined differences in displacements between the medial and lateral menisci. Loads were applied to the joint, and joint load and displacements were measured. Meniscal displacements were measured radiographically. With a joint compressive load of $1 \mathrm{kN}$, internal and external joint rotations caused the lateral meniscus to displace, on average, $0.37 \mathrm{~mm} \mathrm{deg}^{-1}$ in the anterior-posterior direction, while the medial meniscus displaced $0.19 \mathrm{~mm} \mathrm{deg}^{-1}$. Anterior and posterior joint translation, performed under $1 \mathrm{kN}$ joint compression, caused the lateral meniscus to displace, on average, $0.66 \mathrm{~mm} \mathrm{~mm}^{-1}$ in the anterior-posterior direction, while the medial meniscus displaced $0.43 \mathrm{~mm} \mathrm{~mm}^{-1}$. Greater meniscal displacements were found at 15 and $30^{\circ}$ fexion than at $0^{\circ}$ for the lateral meniscus in internal rotation. Lateral meniscal displacements were larger than those of the medial with posterior tibial translation at full extension and with internal rotation.
\end{abstract}

\section{NTRODUCTION}

Injury to a meniscus of the knee is one of the most frequent types of intra-articular injuries. It is widely accepted that loss of a meniscus increases the risk of joint degeneration (Ahmed and Burke, 1983; Fairbank, 1948; Fukubayashi and Kurosawa, 1980; Hsieh and Walker, 1976; Kettlekamp and Jacobs, 1972; Kurosawa et al., 1980; Levy et al., 1982; Seedhom and Hargreaves, 1979; Shoemaker and Markolf, 1986; Tapper and Hoover, 1969; Walker and Erkman, 1975). Meniscal lesions can occur as a result of initial trauma to the joint; in addition, there is a strong correlation between primary anterior cruciate ligament (ACL) injury and subsequent meniscal lesions (Baker et al., 1985; Fox et al., 1979). Arthroscopic repair of meniscal lesions has increased dramatically in recent years, and methods of replacing severely damaged menisci are under investigation (e.g. Arnoczky et al., 1988, 1990).

To prevent joint degeneration and reinjury, the repaired tissue or replacement presumably must possess mechanical characteristics similar to those of the original meniscus. Previous mechanical studies have shown that one of the functions of the meniscus is to distribute contact forces over the articular surfaces by increasing joint contact areas (e.g. Ahmed and Burke, 1983). The knee joint is capable of motion in several

Received in finul form 6 August 1993.

*Author to whom correspondence should be addressed at: Noyes-Giannestras Biomechanics Laboratories, Department of Aerospace Engincering and Engineering Mechanics, University of Cincinnati, Cincinnati, $\mathrm{OH} 45221-0048$, U.S.A. Tel. (513) 556 -4171.

This paper was presented in part at the 32nd and 33rd Annual Meetings of the Orthopaedic Research Society. directions due, in part, to the incongruency of the joint surfaces. Further, the position and curvature of the contact surfaces change with joint position. The menisci resolve the competing requirements of pressure distribution and incongruent joint surfaces by conforming to the articular surfaces and moving as the femur and tibia move.

The menisci are attached to the tibia by powerful insertions into the anterior and posterior intercondylar regions of the tibial spine. They are also attached to the joint capsule peripherally and have other relatively minor restraints. The medial and lateral menisci have distinguishing features. The insertions of the medial meniscus are further apart than those of the lateral. The body of the medial meniscus is both wider and thicker posteriorly than anteriorly; the lateral meniscus is more symmetrical and is wider on average. The radius of curvature of the medial meniscus in the transverse plane is larger than that of the lateral. The anterior horn of the medial meniscus attaches to the anterior ridge of the tibia and the intercondylar eminence. The medial meniscus connects tightly to the joint capsule and the posterior oblique ligament just behind the medial collateral ligament (MCL); also, it often has a connection with the ACL. The transverse ligament connects the anterior portions of the medial and lateral menisci. The horns of the lateral meniscus insert directly into the intercondylar eminence. The ligament of Wrisberg connects the posterior horn of the lateral meniscus to the posterior cruciate ligament (PCL). The lateral meniscus connects only loosely to the joint capsule; just posterior to the lateral collateral ligament (LCL), its attachments to the capsule are interrupted by the tendon sheath of the popliteal tendon. The relative proximity of the lateral meniscus tibial insertions and its looser connections to the 
capsule and ligaments allow the lateral meniscus more mobility than the medial meniscus.

The displacements of the menisci have been described qualitatively by examination of cadaver knees and by arthroscopy (Brantigan and Voshell, 1941; DePalma, 1954; Muller, 1983; Ricklin et al., 1983; Shaer, 1938). These studies noted that (a) flexion caused the menisci to slide posteriorly on the tibial plateau as they followed the contact areas of the femur, (b) flexion-extension occurred mainly between the femur and menisci, (c) internal and external rotation occurred predominantly between the menisci and the tibia, and (d) displacement of the lateral meniscus was more pronounced than that of the medial meniscus. Recently, Thompson et al. (1991) used magnetic resonance imaging to visualize and measure the motions of the menisci in intact cadaver knees. They flexed the joint over its full range with the knee in neutral rotation, with no applied joint loads. While it is widely accepted that meniscal injuries most likely occur during combined loading conditions that include compression, the displacements of the menisci in joints subjected to loads in the physiological range have not been reported.

The purpose of this study was to test several hypotheses regarding the motion of the menisci under joint compression: (1) increased joint compression expands the menisci radially; (2) the addition of internal and external tibial torque displaces the menisci; (3) the addition of anterior and posterior tibial forces translates the menisci; (4) meniscal displacements under a given load increase if the flexion angle is increased from 0 to $30^{\circ}$; and (5) the lateral meniscus displaces further than the medial under any given loading condition.

\section{MATERIALS AND METHODS}

Nine unembalmed human knee joints, including three pairs, were obtained within $48 \mathrm{~h}$ of death and were stored frozen at $-15^{\circ} \mathrm{C}$ prior to testing. The age, sex, and cause of death were not generally known, but the capsules, ligaments, articular cartilage, and menisci were intact, and none had severe degenerative changes. The results of each pair of knees were later averaged, leaving six independent specimens.

The muscles, patella, and fibula with its lateral collateral ligament (LCL) attachment were removed. The remaining ligaments and capsule were left intact. The bones were cut just distal to the tibial insertion of the medial collateral ligament, and just proximal to the collateral ligament insertions on the femur. All trabecular bone was removed from both bones. The hollow cortices were filled with radiolucent polymethylmethacrylate (PMMA) and were cemented into acrylic blocks.

Meniscal displacements were measured radiographically (Fig. 1). Five to seven flat, circular lead markers, $1.5 \mathrm{~mm}$ in diameter and $0.2 \mathrm{~mm}$ thick, each with a small central hole, were cemented with cyan- oacrylate adhesive to the superior surfaces of the menisci. The surfaces were wiped dry and touched with acetone prior to fixing the markers. Two larger markers served as fixed points on the tibial plateau. These were recessed below the surface of the tibial articular cartilage, and were cemented to the subchondral bone. For the purpose of securing the markers to the menisci, the anterior aspect of the medial meniscus was readily accessible from the anterior opening in the capsule, as was the entire lateral meniscus in most knees. Access to the posterior aspect of the medial meniscus was obtained through a $10-20 \mathrm{~mm}$ horizontal incision in the posterior capsule.

The markers were placed around the circumference of the meniscus, usually midway between the inner and peripheral edges of the fibrocartilage. On the posterior aspect of the medial meniscus, the markers were placed closer to the outer edge because of the tightness of the joint in this area. The region near the medial collateral ligament (MCL) was too tight to permit the attachment of a marker. The first and last markers were placed on the fibrocartilage just adjacent to the meniscal horns.

The knee joint loading device was designed to apply quasistatic joint compression in addition to internal and external (IE) torques and anterior and posterior (AP) forces to the tibia, while constraining only those motions of the femur that were necessary to fix the flexion angle and resist the motion of the femur in the directions of whatever loads were applied to the tibia at that time. For example, when an AP force and joint compression were applied to the tibia, three femoral displacements were fixed [flexion-extension (FE) rotation, proximal-distal (PD) translation, and AP translation], while the IE, varus-valgus (VV), and medial-lateral (ML) degrees of freedom of the femur were left free. Tibial PD displacement was always unconstrained for all loading conditions, In addition, the device allowed exposure of transverse radiographs of the knee during loading, while the angle of the tibia with respect to the X-ray source and film remained constant.

The load application device is shown in Fig. 2(a). Forces and torques were applied to the tibia with double-acting pneumatic cylinders and were measured with custom strain gage load cells. The cylinders moved aluminum plates which translated with respect to adjacent plates by means of a system of bearings [Fig. 2(a)]. The AP cylinder moved a plate relative to the stationary laboratory base by means of linear bearings riding on steel shafts. The compression cylinder was fixed to this plate, and so also translated in the AP direction. The tibia was set into a plate which was attached to the top of the compression cylinder and so translated in the PD direction relative to the AP plate, again by means of steel shafts in linear bearings. This tibial plate was composed of an aluminum base and acrylic top. IE torques were applied to the tibial plate by a pair of cylinders attached to moment arms and then to the shaft of the compression cylinder. 


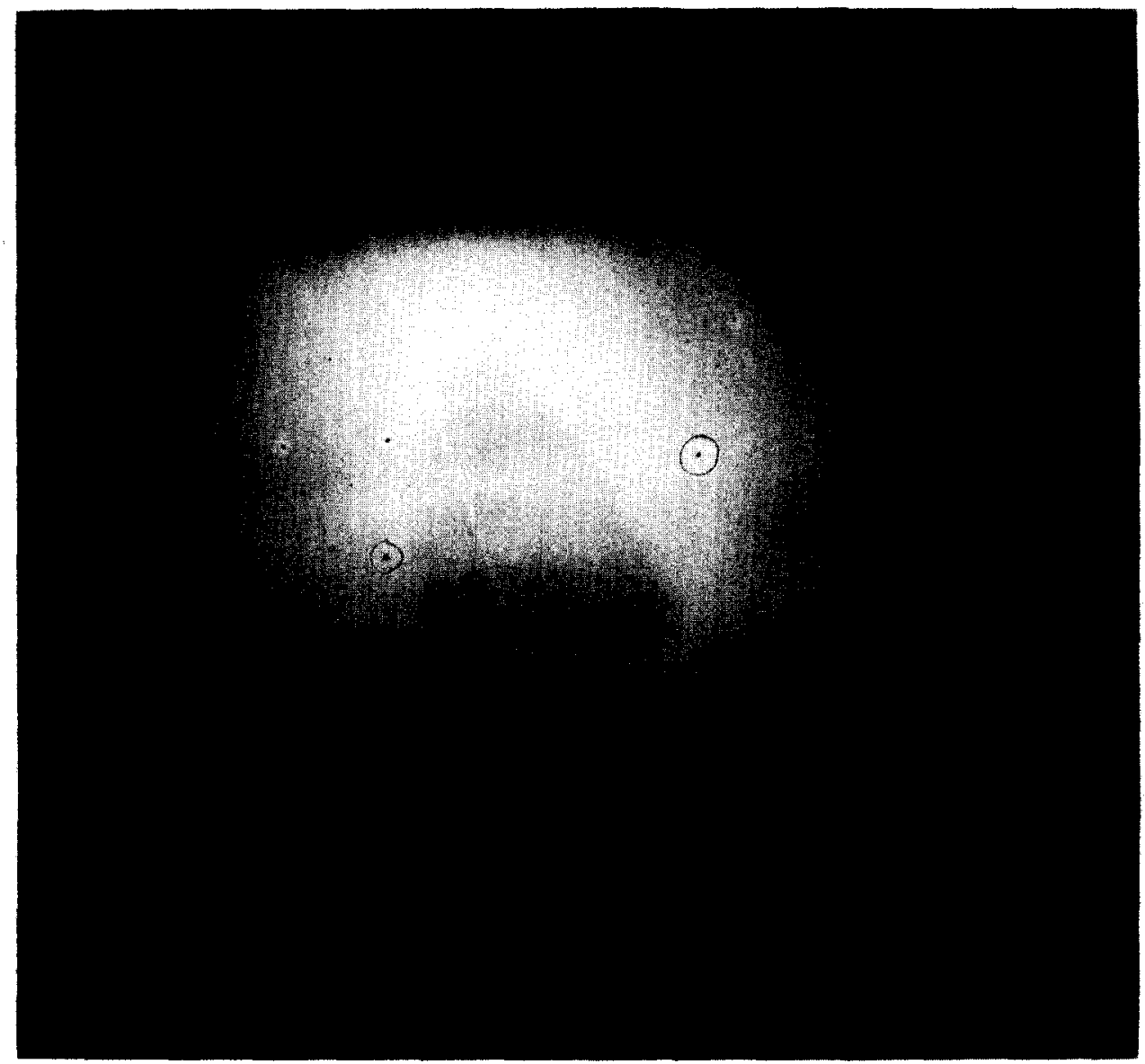

Fig. 1. Transverse radiograph: superior view of left knee, anterior top, medial right. Planar displacements of the menisci with respect to the tibial plateau were measured radiographically. The smaller diameter markers were attached to the superior meniscal surfaces. The two larger markers were recessed below the surface of the tibial articular cartilage and attached to subchondral bone. The horizontal lines across the top and bottom are the tibial axes, the nearly vertical line is one of the femoral axes. 

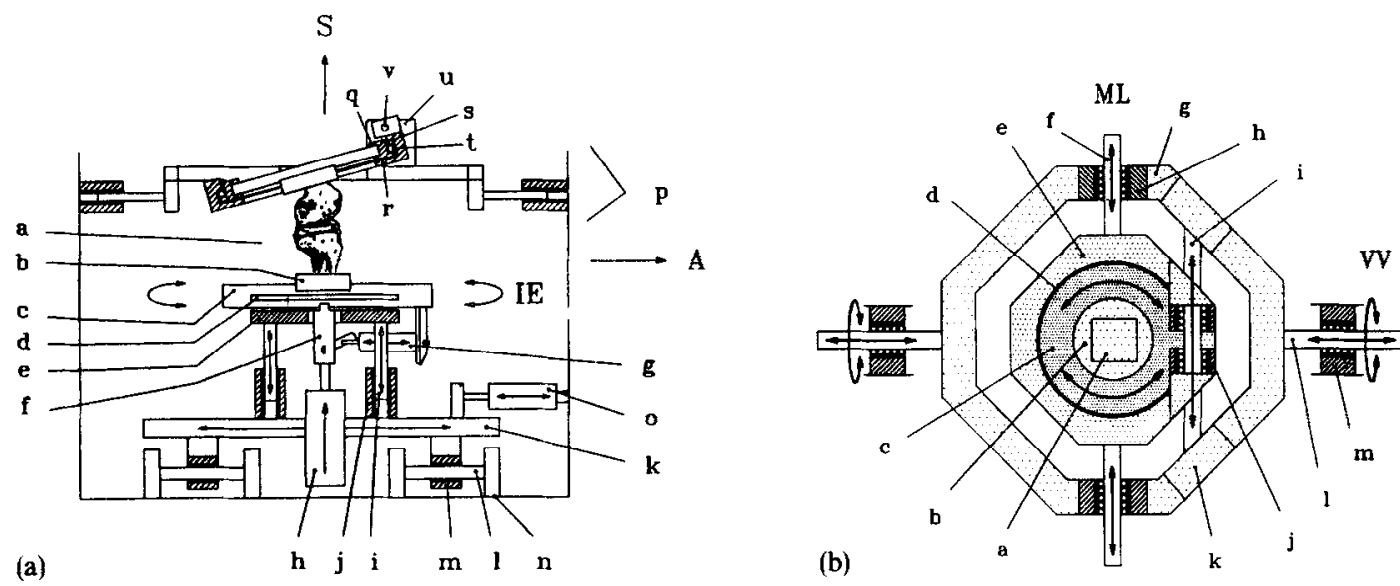

Fig. 2. Schematics of the loading device, 2(a). Combination cross section and side view: the knee specimen (a) is fixed into acrylic block (b) which is press-fit into a matching milled depression in acrylic plate (c). The Xray film fits into slot (d). Plate (c) lies on plate (e), separated by a layer of low-friction plastic which allows for relative IE rotation. Plate (c) is connected through adaptor (f) to pneumatic cylinders which apply joint compression and IE torsion. A pair of cylinders (g) push on moments arms (out of plane of page) to rotate adaptor (f) and plate (c) about the IE axis. Cylinder (h) pushes on plates (e) and (c) to produce compression. Proximal-distal motion is maintained by four shafts (i) in linear bearings (j). These bearings (j) and the compression cylinder (h) are attached to AP plate (k). The AP plate $(k)$ rides on shafts (1) in linear bearings (m). Shafts (1) are attached to the stationary laboratory base (n). Cylinder (o) applies AP forces to plate (k). At the femoral component (p), a cross section of a thrust bearing [see Fig. 2(b)] and the method of fixing the flexion angle are shown. As compression is applied to the joint, the inner section (q) of the thrust bearing lifts off the lower lip of the outer section (r), and so presses against the upper lip (s) through ball bearings ( $t$ ). The flexion angle is fixed by means of blocks of different heights $(u)$ attached with shaft $(v)$ to the outer section of the thrust bearing. 2(b). Top view of the femoral component: anterior right, IE perpendicular to page. The femur is fixed to acrylic block (a), which is press-fit into a matching milled depression under acrylic window (b). Window (b) is fixed to the inner section (c) of a thrust bearing. The race of the thrust bearing (d), which contains $1 / 8$ in diameter ball bearings around its circumference, allows the inner section (c) of the thrust bearing to rotate relative to its outer section (e) about the femoral IE axis. Two case hardened steel shafts (f) connect the thrust bearing to the outer ring (g) of the device. The shafts ride in combination linear and rotary bearings (h), which allow for ML translation and FE rotation of the thrust bearing relative to the outer ring. The flexion angle is fixed by means of shaft (i) which is fixed to the outer ring (g); the thrust bearing is still able to translate in the ML direction, as shaft (i) is held in linear bearings (j) which are attached to the outer section of the thrust bearing (e). The flexion angle is changed by means of blocks of different heights (k). The outer ring ( $\mathrm{g}$ ), then, is attached to two shafts (1). These ride in combination linear and rotary bearings (m) which are fixed to the stationary laboratory base, allowing for AP translation and VV rotation of ring (g) relative to ground.

A relatively radiolucent path was provided through the joint from the X-ray source to the film. This was accomplished by fixing the tibia and femur to two acrylic blocks, as previously noted, which were then press-fit into acrylic plates in the femoral and tibial components of the loading device. The $\mathrm{X}$-ray film was placed in a slot within the tibial acrylic plate. The Xray source was positioned above the femoral acrylic plate. Two sets of coordinate axes made of fine wire were fixed onto the acrylic plates, one set on the bottom surface of the tibial plate adjacent to the $\mathrm{X}$-ray film, and the second on the top surface of the femoral plate. The distances from the tibial plateau to each axis plane were measured to determine the magnification factor at the level of the plateau by linear interpolation in order to find the actual magnitudes of the meniscal displacements.

The femoral component consisted of a combination gimbal, $x-y$ table, and large diameter rotary thrust bearing (RTB), with a maximum of five degrees of freedom [Fig. 2(b)]. One pair of combination linear and rotary bearings permitted AP translation and VV rotation about the AP axis, while another pair permitted free $M L$ translation and FE rotation about the ML axis. During a test, the flexion angle was always fixed. This was accomplished without restricting ML translation by holding the shaft which fixed the flexion angle in linear bearings aligned with the ML direction [Fig. 2(b)]. The femoral acrylic plate was held in the RTB [cross section, Fig. 2(a); top view, Fig. 2(b), which allowed for IE rotation of the femur. The rotation could be locked by means of friction clamps. The AP translation of the femur was locked, using split block shaft collars, only when AP forces were applied to the tibia. Similarly, femoral IE rotation was locked only when IE torques were applied to the tibia. Femoral ML translation and VV rotation were always left free.

Care was taken to fix the joint such that (a) the midpoint of the tibial tubercle and the trochlear 
groove was in line with the AP axis of the device with the joint in neutral rotation, (b) the approximate plane of the tibial plateau was parallel to the X-ray film, (c) the cruciates were centered over the compression cylinder, and (d) the joint was fully extended with the device fixed in full extension.

The stiffness of the device in the IE and AP directions was measured by inserting into the loading device a torque cell which was essentially infinitely stiff compared to the rest of the system, then applying the test loads. The device was found to be stiffer than the joints by more than an order of magnitude in the IE direction; device stiffness in the AP direction was accounted for in the calculation of joint AP displacement. The torque cell was also used to calibrate the strain-gaged moment arms used to measure IE torque.

The test protocol consisted of a sequence of 11 load cases, repeated for each of three flexion angles, 0,15 , and $30^{\circ}$ (Table 1). In order, the load cases were (a) $0.25 \mathrm{kN}$ joint compression, (b) $1 \mathrm{kN}$ joint compression alone, which was then applied in combination with load cases (c)-(j), (c) $100 \mathrm{~N}$ anterior forces, (d) $150 \mathrm{~N}$ anterior force, (e) $100 \mathrm{~N}$ posterior force, (f) $150 \mathrm{~N}$ posterior force, (g) $10 \mathrm{Nm}$ internal torque, (h) $15 \mathrm{Nm}$ internal torque, (i) $10 \mathrm{Nm}$ external torque, (j) $15 \mathrm{Nm}$ external torque, (k) repetition of $1 \mathrm{kN}$ compression alone.

The reference position of the joint was defined as the position assumed by the femur after the slow application of $1 \mathrm{kN}$ compression with the femoral AP, ML, VV, and IE degrees of freedom unlocked [load case (b)]. This reference was chosen for two reasons: joint position is better defined under a significant compressive load because the joint is stiffer, and each of the load cases then differed from the reference condition by just one additional applied load. Combined loading conditions are thought to produce the most menis-

Table 1. Sequence of loads applied for flexion angles of 0,15 , and $30^{\circ}$

\begin{tabular}{llc}
\hline Load case & Direction & Force or torque \\
\hline (a) & Compression & $250 \mathrm{~N}$ \\
(b) & Compression & $1000 \mathrm{~N}$ \\
(c) & Anterior & $100 \mathrm{~N}$ \\
& + compression & $1000 \mathrm{~N}$ \\
(d) & Anterior & $150 \mathrm{~N}$ \\
& + compression & $1000 \mathrm{~N}$ \\
(e) & Postcrior & $100 \mathrm{~N}$ \\
& +compression & $1000 \mathrm{~N}$ \\
(f) & Posterior & $150 \mathrm{~N}$ \\
& +compression & $1000 \mathrm{~N}$ \\
(g) & Internal & $10 \mathrm{~N} \mathrm{~m}$ \\
& + compression & $1000 \mathrm{~N}$ \\
(h) & Internal & $15 \mathrm{~N} \mathrm{~m}$ \\
& +compression & $1000 \mathrm{~N}$ \\
(i) & External & $10 \mathrm{~N} \mathrm{~m}$ \\
& + compression & $1000 \mathrm{~N}$ \\
(j) & External & $15 \mathrm{Nm}$ \\
(k) & tcompression & $1000 \mathrm{~N}$ \\
\hline
\end{tabular}

cal injuries and were therefore of most interest in this study.

Prior to changing the direction of the applied force, all loads and locks were released, the knee was moved to its approximate initial position, and the compressive load was again applied. The femoral displacement in the direction of the AP force or IE torque was then fixed. The AP force or IE torque was then applied to the tibia within a $10 \mathrm{~s}$ time period. Radiographs were taken from above, after equilibrium was reestablished.

Meniscal displacements and IE tibial rotations were measured radiographically by first aligning the position of the fixed markers and axes of the reference load case (b) to each of the other load cases within each flexion angle. The AP and ML components of the displacement of each marker were then measured to the nearest $0.5 \mathrm{~mm}$ and were adjusted by the magnification factor. The mean values of medial and lateral markers for each load case were determined. Spatial averaging was used in order to compare overall meniscal motions by load case, without having to compare displacements of each individual marker. Mean meniscal displacement components were then plotted as a function of joint position, i.e. AP translation or IE rotation, and flexion angle. Joint position was plotted rather than joint load because the correlation between meniscal displacement and joint displacement was stronger than that between meniscal displacement and joint load, as the relationship between joint load and joint displacement is nonlinear (e.g. Markolf et al., 1976; Fukubayashi et al., 1982). Radial expansion due to increasing the compressive load from 250 to $1000 \mathrm{~N}$ was defined by an increase in the distances of all markers toward the joint periphery between load cases (a) and (b).

The X-ray film (Kodak XAR-5) was used without intensifying screens. With the source (Mobile 100 , General Electric) power at $85 \mathrm{kV}_{\mathrm{P}}$ and $15 \mathrm{ma}$ for a duration of $5 \mathrm{~s}$, the central holes in all of the meniscal markers were detectable in most joint positions.

AP translation was measured with a linear potentiometer mounted parallel to the AP pneumatic cylinder. The displacement of the device at the equilibrium load, as calculated from the device stiffness, was subtracted from the measured AP joint displacement.

Statistical tests (SAS, Cary NC) were performed to determine correlation coefficients and linear regression equations for mean meniscal displacement versus joint position. Paired $t$-tests were used to test for (a) differences between medial and lateral meniscal displacements by load case and flexion angle, (b) to determine if the reference joint position was repeatable by comparing the reference and final load cases, and (c) to check for radial expansion of the menisci due to increasing the compression from 250 to $1000 \mathrm{~N}$. Analysis of variance was used to check for difference due to the three flexion angles for the medial and lateral menisci by load case. 


\section{RESULTS}

No radial expansion of the menisci due to increasing joint compression from 250 to $1000 \mathrm{~N}$ was measurable by these radiographic methods. That is, no gross radial displacement was observed due to pure joint compression.
With the addition of external tibial torque to compression of the joint, the lateral meniscus moved anteriorly with respect to the tibial plateau. With an internal torque, the lateral meniscus moved posteriorly. The medial meniscus moved anteriorly with internal rotation and posteriorly with external rotation [Fig. 3 (a)-(c)]. Note that these are relative menis-
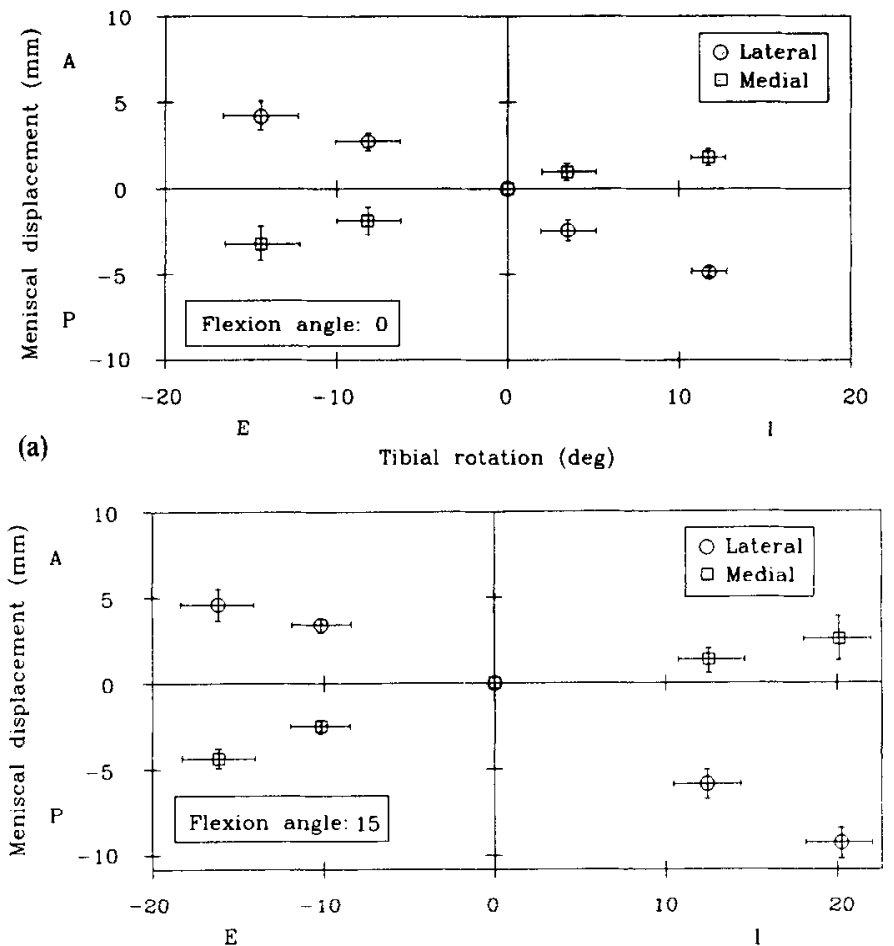

(b)

Tibial rotation $(\mathrm{deg})$

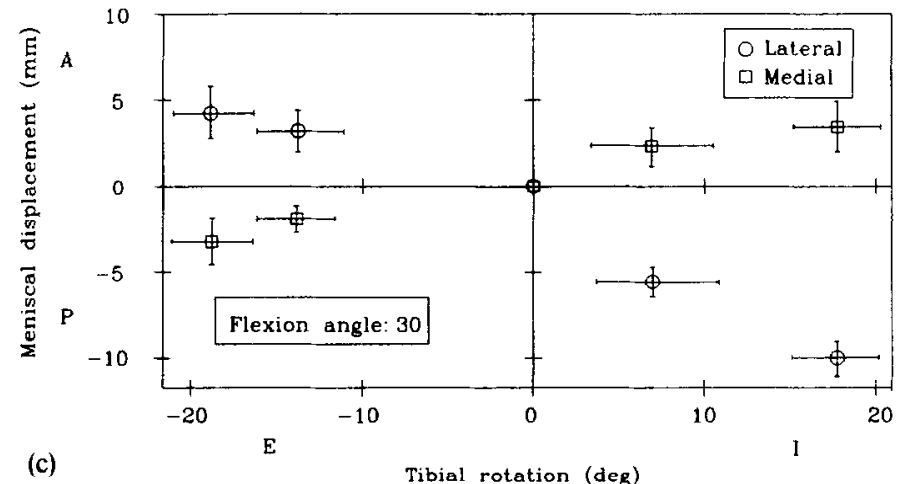

(c)

Tibial rotation (deg)

(d)

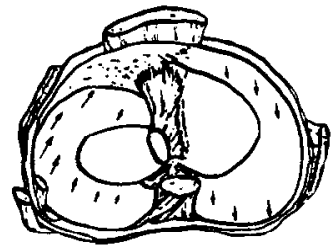

(e)

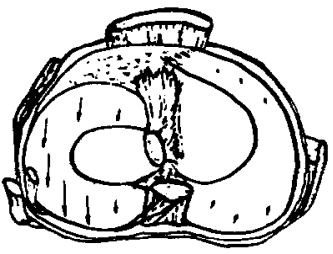

Fig. 3. External tibial rotation ( $\left.E_{3}-\right)$ moved the lateral meniscus anteriorly $(A,+)$; internal tibial rotation $(\mathrm{I},+)$ moved it posteriorly. Mean \pm S.E.M. for $n=6$. Inner data points: $10 \mathrm{Nm}$ applied torque; outer points: $15 \mathrm{Nm}$. (a) $0^{\circ}$ flexion, (b) $15^{\circ}$ flexion, (c) $30^{\circ}$ flexion, (d) and (e): schematics of displacements of all markers for one typical specimen at full extension. Left knee, anterior top, medial right. (d) External rotation, (e) internal rotation. 
cal motions with respect to the tibial plateau; qualitatively, the menisci 'stayed with the femur' as the tibia was moved. Meniscal displacement components in the ML direction were smaller and more random for all loading conditions; therefore, only the AP components of meniscal displacement are reported. Anterior meniscal displacements are denoted as positive, posterior displacements negative; internal tibial rotations are positive, external are negative. Note that because a range of tibial rotations as well as meniscal displacements resulted from applying any particular load level, the means and error bars were used for both the ordinate and abscissa [Figs 3(a)-(c) and 4]. Pictorial views of the meniscal displacement patterns for one representative knee are presented for external rotation [Fig. 3(d)] and internal rotation [Fig. 3(e)].

The relationship between mean AP meniscal displacement components and tibial IE rotation was highly linear for both menisci (Fig. 3; Table 2, r). For the lateral meniscus, the slope of the linear regression line ranged from $-0.34 \mathrm{~mm} \mathrm{deg}^{-1}$ at full extension to $-0.39 \mathrm{~mm} \mathrm{deg}^{-1}$ at $30^{\circ}$ flexion (Table $2, \mathrm{~m}$ ). For the medial meniscus, the slope was $1.9-2.0 \mathrm{~mm} \mathrm{deg}^{-1}$. The probability that the slopes were equal to zero was less than 0.004 for all cases (Table 2, $p$ ).

The effect of increasing flexion from 0 to $30^{\circ}$ on magnitude of meniscal displacements with applied compression plus internal or external torques was suggestive, but usually not statistically significant. Meniscal displacements tended to be greater at 15 and $30^{\circ}$ than at full extension. Significant differences of $4-5 \mathrm{~mm}(p<0.001)$ were found between meniscal displacements at $0^{\circ}$ flexion and those at the higher flexion angles for the lateral meniscus in internal rotation. Also, the magnitude of rotation due to the applied torque and the variability of meniscal displacements increased with flexion from 0 to $30^{\circ}$, reflecting increased joint laxity at $30^{\circ}$. The largest mean meniscal displacements occurred at 30 and $15^{\circ}$ flexion. For the lateral meniscus, these were $-10 \mathrm{~mm}$ for internal

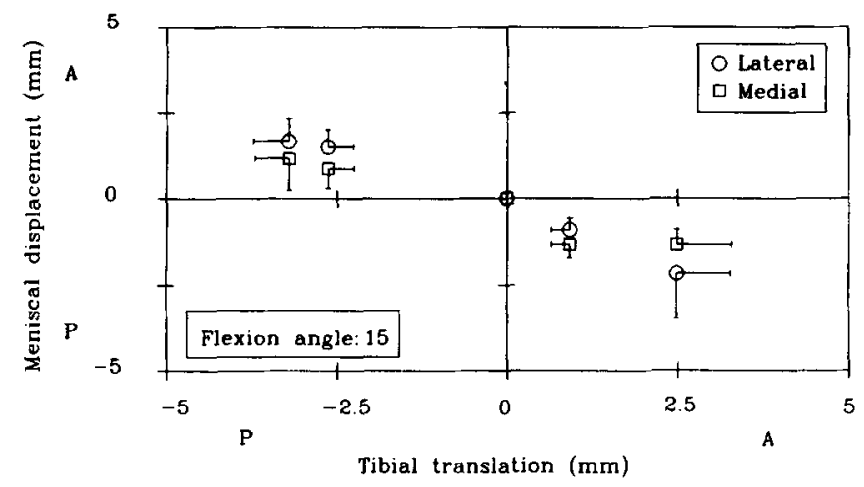

Fig. 4. Anterior tibial translation $(A,+)$ moved the lateral medial menisci posteriorly $(P,-)$; posterior tibial translation moved both menisci anteriorly. Mean \pm S.E.M. for $n=6,15^{\circ}$ flexion. Inner data points correspond to $100 \mathrm{~N}$ applied force, outer points to $150 \mathrm{~N}$.

Table 2. The relationship between AP meniscal displacement components and tibial IE rotation was highly linear for medial and lateral menisci. The relationship between mean AP components of meniscal displacement and tibial AP displacement was also highly linear. Correlation and regression statistics for mean meniscal displacements in the AP direction as a function of (a) internal and external tibial rotation and (b) anterior and posterior tibial translation. Correlation coefficient $(r)$, slope of regression line $(m)$, and probability that the slope of the regression line is zero $(p)$ for lateral and medial menisci, by flexion angle

\begin{tabular}{lcccccc}
\hline & \multicolumn{2}{c}{ Tibial internal-external rotation } & \multicolumn{4}{c}{ Tibial anterior-posterior translation } \\
\hline & $r$ & $m\left(\mathrm{~mm} \mathrm{deg}^{-1}\right)$ & $p$ & $r$ & $m\left(\mathrm{~mm} \mathrm{~mm}^{-1}\right)$ & $p$ \\
\hline Lateral meniscus & & & & & & \\
Flexion angle (deg) & & & & & & \\
0 & -0.99 & -0.34 & 0.001 & -0.97 & -0.68 & 0.007 \\
15 & -0.99 & -0.39 & 0.001 & -0.99 & -0.67 & 0.001 \\
30 & -0.98 & -0.39 & 0.004 & -0.94 & -0.64 & 0.020 \\
Medial meniscus & & & & & & \\
Flexion angle (deg) & & & & & & \\
0 & 0.99 & 0.20 & 0.002 & 0.95 & 0.39 & 0.015 \\
15 & 0.98 & 0.19 & 0.003 & 0.96 & 0.47 & 0.001 \\
30 & 0.99 & 0.19 & 0.002 & 0.97 & 0.43 & 0.006 \\
\hline
\end{tabular}


rotation and $4.5 \mathrm{~mm}$ for external rotation. For the medial meniscus, the largest mean displacements were $3.5 \mathrm{~mm}$ for internal rotation and $-4.5 \mathrm{~mm}$ for external rotation.

The difference between medial and lateral meniscal displacements with applied IE torque was also suggestive, but usually not statistically singificant. While lateral meniscal displacements tended to be larger than those of the medial meniscus, statistically significant differences of $3-7 \mathrm{~mm}(p<0.05)$ were found in the absolute magnitude of medial and lateral meniscal displacements in internal rotation only [load case (i)].

With the addition of anterior tibial force to joint compression, the lateral and medial menisci moved posteriorly, while both moved anteriorly with an added posterior tibial force (Fig. 4). The relationship between the mean AP components of meniscal displacement and tibial AP displacement was linear for both medial and lateral menisci (Fig. 4; Table 2,r). The average slope of the regression lines for the three flexion angles was $0.66 \mathrm{~mm} \mathrm{~mm}^{-1}$; for the medial meniscus, this value was $0.43 \mathrm{~mm} \mathrm{~mm}^{-1}$. The probability that the slopes were equal to zero was less than 0.02 for all cases.

The effect of flexion angle from 0 to $30^{\circ}$ on magnitude of meniscal displacements with applied compression plus anterior or posterior force was not statistically significant, although there was a trend towards greater meniscal mobility with increasing flexion. Displacements at $15^{\circ}$ are shown as an example (Fig. 4).

A small but significant difference of $1 \mathrm{~mm}$ was found between medial and lateral displacements with posterior transiation at full extension only $[p<0.0003$, load case (f), $p<0.05$, load case (e)]. For the lateral meniscus, the largest mean displacements in anterior and posterior tibial translation were -2 and $1.5 \mathrm{~mm}$, respectively. For the medial meniscus, the largest mean displacements were -1.5 and $1 \mathrm{~mm}$, respectively.

The ability to reproduce the joint's neutral position between load cases was verified by comparing meniscal displacements of the reference and last load cases, i.e. the two cases in which pure compression of $1 \mathrm{kN}$ was applied. Displacements of lateral and medial menisci were compared separately by flexion angle. A significant difference $(1.3 \mathrm{~mm})$ was found only for the lateral meniscus at $0^{\circ}(p<0.02)$, indicating that neutral placement sufficient to measure meniscal motion was usually, though not always, repeatable.

No gross observational or statistical difference in meniscal motion between paired joints was found. This supported the decision to average the pairs, which could not be treated as independent specimens.

The magnification factor at the level of the tibial plateau was $1.15 \pm 0.03$ for all specimens combined. The difference between the mean and the extreme magnification factors at the tibial plateau was $\pm 3 \%$; the mean was applied to the meniscal displacements for all specimens.

\section{DISCUSSION}

Meniscal injuries most likely occur due to abnormal meniscal displacements while the joint is subjected to combined loading conditions. Therefore, the methods described were developed to test hypotheses regarding meniscal displacements under simulated physiological loads that included compression, IE torsion, and AP force.

A number of factors limited the accuracy and resolution of these measurements. The menisci were treated as rigid bodies, while they are actually deformable fibrocartilage: they not only displace, but also undergo large strains. With the radiographic method used, meniscal displacements of approximately $\pm 0.5 \mathrm{~mm}$ could be resolved. A further restriction on resolution resulted because displacements were measured by comparing marker locations for each load case to the reference load case, rather than taking $X$ rays before and after each load was applied. The resolution of IE rotation was estimated to be $\pm 0.5^{\circ}$. AP joint position was measured at the AP degree of freedom of the device, rather than at the joint itself. The resolution of AP joint position was estimated to be $\pm 0.5 \mathrm{~mm}$.

Removal of muscle, the patellar mechanism, and especially the LCL, probably eliminated some limits to joint motion and therefore some limits to meniscal motion. The effect of removal of the LCL was judged to be of secondary importance, with the greatest effect in external rotation, based on the results of Ahmed et al. (1987). They reported that the tension patterns of the LCL showed marked variation among specimens; very small tension was generated in IR in $48 \%$ of their specimens and in either sense of rotation in $20 \%$ of their specimens.

Several other factors may have affected the results. The markers themselves, while thin, may have reduced meniscal motion. A few markers sometimes became detached or lost in a dense background, but their locations appeared to be randomly distributed. The sequence of applied loads was fixed, precluding the possibility of separating cumulative damage to the specimen from the effects of increasing the flexion angle. As with all multi-degree-of-freedom loading devices, it was not possible to align the joint precisely within the loading device, or totally preclude the application of 'nonpure' applied loads. The joints were taken from elderly patients who had died of normal causes. The effect of age on meniscal motion is not clear; the supporting structures may be more lax than the general population, thus increasing meniscal motions, while the articular surfaces may be less smooth, thus inhibiting motion. Spatial averaging was used here to compare overall meniscal motion by load case. Other types of studies may need to quantify the motion of a part of a meniscus in a particular location. Adaptation of meniscal tissue over time, for instance, due to scarring at a repair site, would likely affect meniscal displacements depending on the extent and location of the disruption. 
The approximation of meniscal motion as planar was judged to be sufficient to at least an order of magnitude. The only components of meniscal displacement in the proximal-distal direction occurred due to the curvature of the tibial plateaus or compression of the tibia and tibial plate between the meniscal plane and the X-ray film plane. Both these components were small compared to meniscal displacements in the AP-ML plane. Out-of-plane motion of markers was judged to have little effect because the central holes in the markers would not be measurably affected by small marker rotations.

Qualitative descriptions of meniscal motions resulting from flexion and IE rotation have existed for many years. Shaer (1983), Brantigan and Voshell (1941), and DePalma (1954) manually moved dissected cadaver joints to observe meniscal motions. Recent summaries of some early works are contained in Ricklin et al. (1983) and Muller (1983). In particular, it has been reported that during internal rotation, the medial meniscus moved anteriorly while the lateral meniscus moved a considerably greater distance posteriorly. Conversely, during external rotation, the femoral condyles moved the medial meniscus posteriorly and the lateral meniscus a greater distance anteriorly. In this study, internal rotation caused significant differences in displacement between medial and lateral menisci, with the lateral meniscus moving 3-7 mm further posteriorly than the medial meniscus moved anteriorly. Further, this study verified that the relatively large motions observed qualitatively by others with no applied joint compression also occurred with a relatively large compressive load was applied. Although no attempt was made in this study to compare meniscal motions due to AP forces and IE torques combined with a low compressive load versus a high compressive load, one would expect the motions to be somewhat smaller at higher load, since joint stiffness increases with joint compression (Markolf et al., 1976). Nonetheless, relatively large meniscal motions were observed with a joint compressive load of $1 \mathrm{kN}$ in this study.

Quantitatively, Brantigan and Voshell (1941) reported that, on average, the medial and lateral menisci moved 2 and $10 \mathrm{~mm}$, respectively, in the AP direction during a full range of fiexion. DePalma (1954) found that these values were 3 and $9 \mathrm{~mm}$, respectively. Much more recently, Shapeero et al. (1988) documented the change in femorotibial contact points during flexion in intact cadavers knees using ultrafast, cine-computed tomography (CT). However, the contrast between soft tissues and adjacent bone is very limited with CT, so meniscal motions were not directly measured. Thompson et al. (1991) examined cadaveric knees through a full range of flexion using magnetic resonance imaging. They reported that over a passive flexion range of 0 to about $120^{\circ}$, the posterior excursions of the medial and lateral menisci were 5.1 and $11.2 \mathrm{~mm}$, respectively. The testing conditions of this study were different from the others in that: (a) meniscal motions were measured here as a function of joint IE and AP displacement at three discrete flexion angles which did not exceed $30^{\circ}$, not during a full range of flexion at neutral IE rotation and (b) relatively large compressive loads were applied to the joint in this study, in contrast to all other studies. However, the absolute value of meniscal displacements at maximum applied IE torque $(15 \mathrm{~N} \mathrm{~m})$ in the flexed knee were very similar to those measured at full flexion: $10 \mathrm{~mm}$ for the lateral meniscus and $4 \mathrm{~mm}$ for the medial meniscus. These probably represent maximum meniscal displacement values for normal joint displacements.

Compression from $250 \mathrm{~N}$ to $1 \mathrm{kN}$ did not cause measurable meniscal radial displacement. This may be surprising in light of free-body diagrams of the meniscus under load (e.g. Fairbank, 1948; Shrive et al., 1978), which show the meniscus expanding in the radial direction as the joint is compressed. The menisci strained radially as well as circumferentially with these levels of joint compression, but they did not displace significantly. Under pure joint compression, they were already in place to bear load, and they were too stiff to expand radially to a degree measurable by these methods. However, it is possible that most expansion occurred from 0 to $250 \mathrm{~N}$ due to the separation of the articular surfaces by the menisci at low load (e.g. Shrive et al., 1978), and to the stiffening characteristics of the material properties of the menisci (e.g. Proctor et al., 1989).

The change in position of the menisci will directly affect previous estimates of the location and magnitude of high stress areas on the menisci and articular surfaces of the tibia and femur. After measuring contact stress in knees subject to a variety of loading conditions, Ahmed et al. (1983) determined the percentage of the load borne by the menisci. These percentages were based on the position of the menisci in the unloaded, neutrally positioned joint. Based on the current study which showed how the menisci move to accomodate the changing relative positions of the articulating tibial and femoral condyles, an even better prediction of the proportions of compartmental load borne by the menisci might be calculated. Further, mathematical models of the knee joint with the menisci which have been reported to date (e.g. Tisshakht et al., 1992) have not taken into account the motion of the menisci with respect to the femur and tibia under combined loading conditions.

Meniscal displacements under different joint loading conditions have been presented. Previous descriptions of the motion of the menisci were verified and quantified. The methods and results presented here may be used to test for the effects of ligament injury, meniscal repair, partial meniscectomy, or meniscal replacement on meniscal motion. It is expected that ligament injury would allow for increased motion of a meniscus, while repair or replacement might allow either too much or too little meniscal motion depending on the materials used and the technique of fixation. Increased or decreased displacement would 
presumably increase the risk of subsequent meniscal injury.

Acknowledgement - Support for this project was provided by The University of Michigan Horace $\mathbf{H}$. Rackham School of graduate studies.

\section{REFERENCES}

Ahmed, A. M. and Burke, D. L. (1983) In-vitro measurement of static pressure distribution in synovial joints. Part I: tibial surface of the knee. J. biomech. Engng 105, 216-225.

Ahmed, A. M., Hyder, A., Burke, D. L. and Chan, H. K. (1987) In vitro ligament tension patterns in the flexed knee in passive loading. J. orthop. Res. 5, 217-230.

Arnoczky, S. P., McDevitt, C. A., Schmidt, M. B., Mow, V. C. and Warren, R. F. (1988) The effect of cryopreservation on canine menisci. J. orthop. Res. 6, 1-12.

Arnoczky, S. P., Warren, R. F. and McDevitt, C. A. (1990) Meniscal replacement using a cryopreserved allograft. An experimental study in the dog. Clin. orthop. Rel. Res. 252, 121-128.

Baker, B. E., Peckham, A. C., Pupparo, F. and Sanborn, J. C. (1985) Review of meniscal injury and associated sports. Am. J. Sports Med. 13, 1-4.

Brantigan, O. C. and Voshell, A. E. (1941) The mechanics of the ligaments and menisci of the knee joint. J. Bone Jt Surg. 23, 44-66.

DePalma, A. F. (1954) Diseases of the Knee. Lippincott, Philadelphia .

Fairbank, T. J. (1948) Knee joint changes after meniscectomy. J. Bone Jt Surg. (Br.) 30B, 664-670.

Fox, J. M., Blazina, M. E. and Carlson G. J. (1979) Multiphasic view of medial meniscectomy. Am. J. Sports Med. 7, 161-164.

Fukubayashi, T. and Kurosawa, H. (1980) The contact area and pressure distribution pattern of the knee. A study of normal and osteoarthrotic knee joints. Acta. orthop. Scand. 51, 871-879.

Fukubayashi, T., Torzilli, P. A., Sherman, M. F. and Warren, R. F. (1982) An in vitro biomechanical evaluation of anterior-posterior motion of the knee. J. Bone Jt Surg (Am.) 64A, 258-264.

Hsieh, H. and Walker, P. S. (1976) Stabilizing mechanisms of the loaded and unloaded knee joint. J. Bone Jt Surg. (Am.) 58A, 87-93.

Kettlekamp, D. B. and Jacobs, A. W. (1972) Tibiofemoral contact area-determination and implication. $J$ Bone $J t$ Surg. (Am.) 54A, 349-356.

Kurosawa, H., Fukubayashi, T. and Nakajima, H. (1980) Load-bearing mode of the knee joint: physical behavior of the knee joint with or without menisci. Clin orthop. Rel. Res. 149, 283-290.

Levy, I. M., Torzilli, P. A. and Warren, R. F. (1982) The effect of medial meniscectomy on anterior-posterior motion of the knee. $J$ Bone Jt Surg (Am.) 64A, 883-888.

Markolf, K. L., Mensch, J. S. and Amstutz, H. C. (1976) Stiffness and laxity of the knee-the contributions of the supporting structures. J. Bone Jt Surg. (Am.) 58A, 583-594.

Muller, W. (1983) The Knee: Form, Function, and Ligament Reconstruction. Springer, Berlin.

Proctor, C. S., Schmidt, M. B., Whipple, R. R., Kelly, M. A. and Mow, V. C. (1989) Material properties of the normal medial bovine meniscus. J. orthop. Res. 7, 771-782.

Ricklin, P., Ruttimann, A. and Del Buono, M. S. (1983) The Meniscus. Diagnosis, Differential Diagnosis and Therapy (2nd Edn). Georg Thieme, Stuttgart.

Seedhom, B. B. and Hargreaves, D. J. (1979) Transmission of the load in the knee joint with special reference to the role of the menisci. Engng Med. 8, 207-228.

Shaer, H. (1938) Der Meniskusschaden. Georg Thieme, Leipzig.

Shapeero, L. G., Dye, S. F and Lipton, M. J. (1988) Functional dynamics of the knee joint by ultrafast, cine-CT. Invest. Radiol. 23, 118-123.

Shoemaker, S. C. and Markolf, K. L. (1986) The role of the meniscus in the anterior-posterior stability of the loaded anterior cruciate-deficient knee. $J$. Bone $J t$ Surg. (Am.) 68A, 71-79.

Shrive, N. G., O' Connor, J. J. and Goodfellow, J.W. (1978) Load-bearing in the knee joint. Clin. orthop. Rel. Res. 131, 279-287.

Tapper, E. M. and Hoover, N. W. (1969) Late results after meniscectomy. J. Bone Jt Surg. (Am.) 51A, 517-526.

Thompson, W. O., Thaete, F. L., Fu, F. H. and Dye, S. F. (1991) Tibial meniscal dynamics using three-dimensional reconstruction of magnetic resonance images. Am. J. Sports Med. 19, 210-216.

Tissakht, M., Ahmed, A. M. and Misra, A. K. (1992) Meniscal response to $3 \mathrm{D}$ complex loadings using a finite element model of the intact tibiofemoral joint. Proc. 38th Annual Meeting, p. 628. Orthopaedic Research Society, Washington, DC.

Walker, P. S. and Erkman, M. J. (1975) The role of the menisci in force transmission across the knee. Clin. orthop. Rel. Res. 109, 184-192. 